despite changes of ulcers or stricturing, biopsies showed focal active ulceration which fell short for the histological criteria for Crohn's disease. There was no change in management in 3 patients due to symptom resolution. On logistic regression, hypoalbuminaemia $(p=0.059)$ and diarrhoea $(p=0.058)$ were predictive of a positive diagnosis on CE. The diagnosis of IBDU remained in $59 \%$ of patients $(n=21)$.

Conclusion CE is useful for identifying patients classed as IBDU who may have underlying Crohn's disease with a positive impact on their management. Further larger studies are required to validate these results.

Competing interests None.

Keywords capsule endoscopy, Crohn's disease, indeterminate colitis.

\title{
PTU-064 $\downarrow$ CAN WE CATEGORISE PATIENTS WITH INFLAMMATORY BOWEL DISEASE UNCLASSIFIED (IBDU) WIRELESSLY?
}

doi:10.1136/gut.2011.239301.192

R Kalla, ${ }_{1}{ }^{*}$ M E McAlindon, R Sidhu Department of Gastroenterology, Royal Hallamshire Hospital, Sheffield, UK

Introduction Capsule endoscopy (CE) has developed an established role in the investigation of patients with Crohn's disease. However, there remains a paucity of published literature on the use of $\mathrm{CE}$ in the setting of patients with indeterminate colitis (IBDU). We investigated the diagnostic yield of CE in patients with (IBDU) and impact on subsequent diagnosis and management.

Methods Patients referred to the CE service routinely from 2003 to 2009 with a diagnosis of IBDU, were identified retrospectively. Data was collected for patient demographics, previous investigations, medications and findings at CE. Patients with findings of three or more ulcers with the presence of erythema and oedema were classed as Crohn's disease. Follow up data was collected on all patients to assess the impact of CE on final outcomes.

Results A total of thirty seven patients with histological indeterminate colitis were identified. There were $78 \%$ females with median age of 47 years (range 18-81 years). The majority of patients $(78 \%)$ had symptoms of diarrhoea. Abdominal pain represented $60 \%$ while $78 \%$ of patients $(n=29)$ had evidence of raised inflammatory markers. All patients had prior colonoscopy with terminal ileal (TI) intubation in $65 \%(n=24)$. Forty two percent of patients $(n=10 / 24)$ had macroscopic abnormalities at TI, of which $10 \%$ were normal and 90\% ( $n=9)$ showed non specific changes histologically. The mean follow up period was 19 months $( \pm 2)$. Small bowel changes including ulceration or erosions, oedema, petechiae and erythema were identified in $43 \%(n=16 / 37)$ of patients on CE. There were no complications with CE. The diagnosis of Crohn's was made in 14 patients at follow up (41\%) with 1 patient lost to follow up and one patient with a diagnosis of non-steroidal ant-inflammatory drug (NSAID) enteropathy. Seven of these patients had a normal small bowel radiology prior to CE. Management was altered in $78 \%$ of patients ( $n=11 / 14)$. Two patients had medication change; steroids $(n=1)$ and anti-TNF $(n=1)$ and histological verification with a repeat ileo-colonoscopy or double-balloon enteroscopy was performed in nine patients. While in two patients, Crohn's disease was confirmed, in seven patients, 